\title{
A Novel Simulation Model for Nonstationary Rice Fading Channels
}

\author{
Kaili Jiang, ${ }^{1}$ Xiaomin Chen $\left(\mathbb{D},{ }^{1}\right.$ Qiuming Zhu $\left(\mathbb{D},{ }^{1,2}\right.$ Lele Chen, \\ Dazhuan Xu, ${ }^{1}$ and Bing Chen ${ }^{1}{ }^{1}$ \\ ${ }^{1}$ Jiangsu Key Laboratory of Internet of Things and Control Technologies, Nanjing University of Aeronautics and Astronautics, \\ Nanjing 211106, China \\ ${ }^{2}$ Institute of Sensors, Signals and Systems, School of Engineering \& Physical Sciences, Heriot-Watt University, \\ Edinburgh EH14 4AS, UK
}

Correspondence should be addressed to Qiuming Zhu; zhuqiuming@nuaa.edu.cn

Received 28 September 2017; Revised 7 December 2017; Accepted 19 December 2017; Published 18 January 2018

Academic Editor: Carlos A Gutierrez

Copyright (C) 2018 Kaili Jiang et al. This is an open access article distributed under the Creative Commons Attribution License, which permits unrestricted use, distribution, and reproduction in any medium, provided the original work is properly cited.

\begin{abstract}
In this paper, we propose a new simulator for nonstationary Rice fading channels under nonisotropic scattering scenarios, as well as the improved computation method of simulation parameters. The new simulator can also be applied on generating Rayleigh fading channels by adjusting parameters. The proposed simulator takes into account the smooth transition of fading phases between the adjacent channel states. The time-variant statistical properties of the proposed simulator, that is, the probability density functions (PDFs) of envelope and phase, autocorrelation function (ACF), and Doppler power spectrum density (DPSD), are also analyzed and derived. Simulation results have demonstrated that our proposed simulator provides good approximation on the statistical properties with the corresponding theoretical ones, which indicates its usefulness for the performance evaluation and validation of the wireless communication systems under nonstationary and nonisotropic scenarios.
\end{abstract}

\section{Introduction}

The channel simulator has the ability to reproduce the statistical properties of propagation channels and has become a very important software-assisted tool for the performance evaluation of wireless communication systems. There are a number of research papers on designing accurate and efficient simulators [1-19]. For the good fitting with plan-wave propagation channels, the sum-of-sinusoids (SoS) $[1,2]$ or sum-ofcisoids ( $\mathrm{SoC}$ ) [3] based simulators and their derivatives [4-8] have gained the most widespread acceptance.

It should be highlighted that the traditional SoC simulators are only suitable to reproduce wide-sense stationary (WSS) fading channels, which means the statistical properties of output channels are time-invariant. However, measurement campaigns have proved that the WSS assumption is only valid for a short time interval [20]. Therefore, several modified SoC-based simulators were proposed in [9-19] to generate nonstationary fading channels. For example, the WINNER+ model [9] was simulated by generating several independent channel segments with different simulation parameters. Simulation parameters were updated according to the fixed trajectories of transceivers and clusters in $[10,11$, $13,14]$, which improves the continuity of adjacent channel states.

However, we have found that the generated channel phases of these simulators in [9-11, 13, 14] cannot guarantee a smooth transition between adjacent channel states, which makes the output Doppler frequency shifts not very accurate. To overcome this shortcoming, a new method, namely, sum of frequency modulation signals [12,15-18] or sum of chirp signals [19], was proposed to simulate nonstationary Rayleigh channels very recently, but it lacked implementation details and performance analyses. To fill this gap, this paper develops a new simulator based on this idea to generate the nonstationary Rice fading channels under nonisotropic scattering scenarios, as well as the upgraded computation methods of simulation parameters. Moreover, the timevariant statistical properties of the proposed simulator, that is, the probability density functions (PDFs) of the envelope 
and phase, autocorrelation function (ACF), and Doppler power spectral density (DPSD), are derived in detail and also verified by numerical simulations.

The rest of this paper is organized as follows. In Section 2, we introduce the reference model and traditional SoC simulators for stationary fading channels. Section 3 proposes a new simulator for the nonstationary Rice channels, as well as computation methods of the time-variant simulation parameters. The theoretical results of PDF, ACF, and DPSD for the proposed simulator are derived in detail in Section 4. In Section 5, simulation results are given and compared with the corresponding derivation results. Finally, conclusions are drawn in Section 6.

\section{Reference and Simulation Models for Stationary Fading Channels}

Under the nonstationary condition, the reference model for Rice fading channels can be written as [3]

$$
v(t)=\sqrt{\frac{\Omega_{v}}{K+1}} \mu(t)+\sqrt{\frac{K \Omega_{v}}{K+1}} m(t),
$$

where $\Omega_{v}$ and $K$ are the mean power and Rice factor, and the non-line-of-sight (NLOS) component $\mu(t)$ denotes a normalized zero-mean complex Gaussian process. In (1), $m(t)=\exp \left(j\left(2 \pi f_{\rho} t+\theta_{\rho}\right)\right)$ represents the line-of-sight (LOS) component, where $f_{\rho}$ and $\theta_{\rho}$ are the Doppler frequency and phase of the LOS component, respectively. From (1), we can easily obtain the reference model for Rayleigh fading channels by omitting the LOS component $(K=0)$.

The characteristics of reference model are determined by its first- and second-order statistical properties, that is, PDF, ACF, and DPSD. Given the envelope $\xi(t) \triangleq|v(t)|$ and phase $\vartheta(t) \triangleq \arg \{v(t)\}$, the reference PDFs can be expressed as [3]

$$
\begin{aligned}
& p_{\xi}(z)=\frac{2 z(K+1)}{\Omega_{v}} e^{-z^{2}(K+1) / \Omega_{v}-K} \\
& \cdot I_{0}\left(\frac{2 z}{\sqrt{\Omega_{v}}} \sqrt{K(K+1)}\right), \quad z \geq 0 \\
& p_{\vartheta}(\theta)=\frac{e^{-K}}{2 \pi}\left\{\left[1+\operatorname{erf}\left(\sqrt{K} \cos \left(\theta-2 \pi f_{\rho} t-\theta_{\rho}\right)\right)\right]\right. \\
&\left.\cdot \sqrt{K \pi} \cos \left(\theta-2 \pi f_{\rho} t-\theta_{\rho}\right) e^{K \cos ^{2}\left(\theta-2 \pi f_{\rho} t-\theta_{\rho}\right)}+1\right\} \\
& \quad 0 \leq \theta<2 \pi,
\end{aligned}
$$

where $I_{0}(\cdot)$ and $\operatorname{erf}(\cdot)$ denote the modified Bessel function of the first kind of order zero and complementary error function, respectively. The ACF of $v(t)$ is defined by $r_{v v}(\tau)=$ $E\left\{v^{*}(t) v(t+\tau)\right\}$, where $E\{\cdot\}$ and $(\cdot)^{*}$ denote the statistical expectation and complex conjugation operator, respectively. Finally, the DPSD can be obtained by the Fourier transform of ACF as $S_{v v}(f)=\int_{-\infty}^{\infty} r_{v v}(t, \tau) e^{-j 2 \pi f \tau} d \tau$. For nonisotropic scattering scenarios, the von Mises (VM) distribution is widely accepted to describe the angle of arrival (AoA) [8] and is given by

$$
p_{\alpha}(\alpha)=\frac{e^{\kappa \cos \left(\alpha-m_{\alpha}\right)}}{2 \pi I_{0}(\kappa)}
$$

where $\kappa>0$ controls the angular spread and $m_{\alpha} \in(-\pi, \pi]$ denotes the mean value. Under the stationary VM scattering scenario, the references ACF and DPSD have been derived as [8]

$$
\begin{aligned}
& r_{v v}(\tau) \\
& =\frac{\Omega_{v} I_{0}\left(\sqrt{\kappa^{2}-\left(2 \pi f_{\max } \tau\right)^{2}+j 4 \pi \kappa f_{\max } \cos \left(m_{\alpha}\right) \tau}\right)}{(K+1) I_{0}(\kappa)} \\
& \quad+\frac{K \Omega_{v}}{K+1} e^{j 2 \pi f_{\rho} \tau}, \\
& S_{v v}(f) \\
& =\frac{\Omega_{v} \operatorname{rect}(f) \cosh \left(\kappa \sin \left(m_{\alpha}\right) \sqrt{1-\left(f / f_{\max }\right)^{2}}\right) e^{\kappa \cos \left(m_{\alpha}\right) f / f_{\max }}}{\pi f_{\max }(K+1) I_{0}(\kappa) \sqrt{1-\left(f / f_{\max }\right)^{2}}} \\
& \quad+\frac{K \Omega_{v} \delta\left(f-f_{\rho}\right)}{K+1},
\end{aligned}
$$

where $f_{\max }=v f_{c} / c$ denotes the maximum Doppler frequency and $v, c$, and $f_{c}$ are the movement speed, light speed, and carrier frequency, respectively.

The traditional SoC simulator can be expressed as [8]

$$
\begin{aligned}
\widehat{v}(t)= & \sqrt{\frac{\Omega_{v}}{K+1} \underbrace{\sum_{\mu}(t)}_{\widehat{n}=1} \sqrt{\frac{1}{N}} e^{j\left(2 \pi \widehat{f}_{n} t+\widehat{\theta}_{n}\right)}} \\
& +\sqrt{\frac{K \Omega_{v}}{K+1}} \underbrace{e^{j\left(2 \pi \widehat{f}_{\rho} t+\widehat{\theta}_{\rho}\right)}}_{\widehat{m}(t)},
\end{aligned}
$$

where $N$ is the number of simulation paths, $\widehat{f}_{n}, \widehat{f}_{\rho}, \widehat{\theta}_{n}$, and $\hat{\theta}_{\rho}$ are the time-invariant simulation parameters, mean Doppler frequencies, initial phases of the NLOS, and LOS component, respectively. Note that the frequency parameters $\widehat{f}_{n}, n=$ $1, \ldots, N$ and $\widehat{f}_{\rho}$ are determined by the ACF or DPSD of the reference model, while the phase $\widehat{\theta}_{n}, n=1, \ldots, N$ and $\hat{\theta}_{\rho}$ are generated randomly and uniformly over $(0,2 \pi]$.

\section{A New Simulator for Nonstationary Fading Channels}

Under the nonstationary condition, the statistical properties of channels would change over time [20], and this makes channel parameters time-variant. In order to apply the classic SoC method, some modified simulators [9-11, 13, 14] directly use $2 \pi f_{n}(t) t$ and $2 \pi f_{\rho}(t) t$ to substitute $2 \pi f_{n} t$ and $2 \pi f_{\rho} t$ in (7), respectively. However, it can be proved that the output phases of these models are not accurate and the output Doppler frequencies do not agree with the theoretical ones [18]. In this 
paper, we use $2 \pi \int_{0}^{t} f_{n}\left(t^{\prime}\right) \mathrm{d} t^{\prime}$ and $2 \pi \int_{0}^{t} f_{\rho}\left(t^{\prime}\right) \mathrm{d} t^{\prime}$ to replace $2 \pi f_{n}(t) t$ and $2 \pi f_{\rho}(t) t$, respectively, and the new simulator can be written as

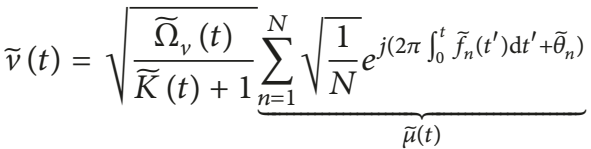

$$
\begin{aligned}
& +\sqrt{\frac{\widetilde{K}(t) \widetilde{\Omega}_{v}(t)}{\widetilde{K}(t)+1}} \underbrace{j\left(2 \pi \int_{0}^{t} \tilde{f}_{\rho}\left(t^{\prime}\right) \mathrm{d} t^{\prime}+\tilde{\theta}_{\rho}\right)}_{\widetilde{m}(t)} .
\end{aligned}
$$

A key issue for the proposed simulator is to find a proper set of simulation parameters $\left\{\tilde{f}_{\rho}, \tilde{f}_{n}\right\}, n=1, \ldots, N$, which can guarantee that the output statistical properties are close to the desired ones. Several parameter computation methods have been proposed in the literature $[8,21-23]$, such as the Riemann sum method (RSM) [8], the extended method of exact Doppler spread (EMEDS) [21], the generalized method of equal areas (GMEA) [22], and the $L_{p}$-norm method [23]. However, these methods are only suitable for the timeinvariant parametric simulator. Therefore, an upgraded computation method is required to make the proposed simulator realizable.

Since the WSS assumption is still valid for the nonstationary channels during a short time interval [20], it is reasonable to assume that the statistical properties keep unchanged during a short time duration $T_{u}$, namely, the updating interval of channel states. Thus, the parameters $\widetilde{\Omega}_{p}(t)$ and $\widetilde{K}(t)$ within the $l$ th interval can be denoted as $\widetilde{\Omega}_{v}^{l}$ and $\widetilde{K}^{l}$, where $l=$ $\left\lfloor t / T_{u}\right\rfloor+1(l=1,2,3, \ldots)$ and $\lfloor\cdot\rfloor$ means the integer part. The corresponding DPSD within the $l$ th interval can be defined by

$$
S_{\widetilde{v v}}^{l}(f)=\frac{\widetilde{\Omega}_{v}^{l}}{\widetilde{K}^{l}+1} S_{\widetilde{\mu} \tilde{\mu}}^{l}(f)+\frac{\widetilde{K}^{l} \widetilde{\Omega}_{v}^{l}}{\widetilde{K}^{l}+1} S_{\widetilde{m} \widetilde{m}}^{l}(f),
$$

where $S_{\widetilde{\mu} \tilde{\mu}}^{l}(f)$ and $S_{\widetilde{m} \widetilde{m}}^{l}(f)$ are the DPSDs of the NLOS component and LOS component, respectively, and can be obtained by (6) under the VM scattering scenario or measured under other realistic scenarios. Then, by using aforementioned methods [8,21-23], the frequency parameters of the $l$ th interval, denoted as $\left\{\widetilde{F}_{\rho}^{l}, \widetilde{F}_{n}^{l}\right\}, n=1, \ldots, N$, can be calculated. Hence, the time-variant frequency parameters can be computed by $\tilde{f}_{\rho}(t)=\widetilde{F}_{\rho}^{l}$ and $\tilde{f}_{n}(t)=\widetilde{F}_{n}^{l}$. Although this computation method is straightforward and has been applied to simulate WINNER+ model [9] and 3GPP-3D model [24], it does not consider the fact that channel properties in reality usually change smoothly along time. On the other hand, measurement campaigns [20] have shown that the stationary interval is very short, that is, $9 \mathrm{~ms}$ in $80 \%$ of the case and $20 \mathrm{~ms}$ in $60 \%$ of the case under the high speed train (HST) scenario. In this paper, we assume that the Doppler frequency changes linearly within the stationary interval, so $\tilde{f}_{\rho}(t)$ can be calculated by

$$
\widetilde{f}_{\rho}(t)=\widetilde{F}_{\rho}^{l}+\frac{\widetilde{F}_{\rho}^{l+1}-\widetilde{F}_{\rho}^{l}}{T_{u}}\left[t-(l-1) T_{u}\right] .
$$

Taking the fact into account that the simulations of MIMO channels often demand for generating multiple uncorrelated Rayleigh or Rice fading waveforms, the new calculation method of $\widetilde{f}_{n}(t)$ is given as

$$
\tilde{f}_{n}(t)=b_{n}^{l}+k_{n}^{l}\left[t-(l-1) T_{u}\right]+\Delta_{n}^{l}(t),
$$

where $b_{n}^{l}, k_{n}^{l}$, and $\Delta_{n}^{l}(t)$ denote the initial value, the slope, and the small random offset of the frequency parameter of the $n$th path, respectively. $b_{n}^{l}(l=1)$ is generated randomly and uniformly over $\left[\widetilde{F}_{n-1}^{1}, \widetilde{F}_{n}^{1}\right)$, while $b_{n}^{l}(l>1)$ holds the value at the end of the previous interval. The slope $k_{n}^{l}$ is calculated by

$$
= \begin{cases}k_{s f}^{l} \frac{\left(\widetilde{F}_{n}^{l}-\widetilde{F}_{n-1}^{l}\right)+\left(\widetilde{F}_{n}^{l+1}-\widetilde{F}_{n-1}^{l+1}\right)}{2 T_{u}}, & \tilde{f}_{n}(t)<B_{n}^{\text {low }}(t) \\ N_{s f} \frac{\left(\widetilde{F}_{n-1}^{l}-\widetilde{F}_{n}^{l}\right)+\left(\widetilde{F}_{n-1}^{l+1}-\widetilde{F}_{n}^{l+1}\right)}{2 T_{u}}, & \tilde{f}_{n}(t)>B_{n}^{\text {upper }}(t),\end{cases}
$$

where $N_{s f}$ refers to the number of period within each interval, and the upper boundary $B_{n}^{\text {upper }}(t)$ and low boundary $B_{n}^{\text {low }}(t)$ are used to keep the frequencies changing within valid ranges, which are defined by

$$
\begin{aligned}
B_{n}^{\text {upper }}(t) & =\widetilde{F}_{n}^{l}+\frac{\left(\widetilde{F}_{n}^{l+1}-\widetilde{F}_{n}^{l}\right)\left(t-(l-1) T_{u}\right)}{T_{u}} \\
B_{n}^{\text {low }}(t) & =\widetilde{F}_{n-1}^{l}+\frac{\left(\widetilde{F}_{n-1}^{l+1}-\widetilde{F}_{n-1}^{l}\right)\left(t-(l-1) T_{u}\right)}{T_{u}} .
\end{aligned}
$$

Note that the parameter computation method in [19] can be viewed as a special case of (11) with $N_{s f}=1$. To visualize and demonstrate the proposed computation method, let us consider the reference DPSDs of $S_{\widetilde{\mu} \tilde{u}}(t, f)$ and $S_{\widetilde{m} \widetilde{m}}(t, f)$ under VM scattering environment as shown in Figure 1. The parameters are configured as follows, $T_{u}=10 \mathrm{~ms}, N=8$, and $N_{s f}=2$. The frequencies and boundaries can be calculated by using (10)-(13) and are also shown in Figure 1. It is clear that the frequencies are continuous and change linearly within the upper and lower boundaries. The frequencies also include a small and random offset, which is to guarantee the generated multiple fading channels are uncorrelated.

\section{Time-Variant Statistical Properties of the Proposed Simulator}

4.1. The PDFs of Envelope and Phase. In order to derive the PDFs of envelope and phase for the proposed simulator, let 
us set $\widetilde{v}(t)=\widetilde{v}_{1}(t)+j \widetilde{v}_{2}(t)$, where $\widetilde{v}_{1}(t)$ and $\widetilde{v}_{2}(t)$ are defined by

$$
\begin{aligned}
& \widetilde{v}_{1}(t) \\
& =\underbrace{\sqrt{\frac{\widetilde{K}(t) \widetilde{\Omega}_{v}(t)}{\widetilde{K}(t)+1}} \cos \left(2 \pi \int_{0}^{t} \tilde{f}_{\rho}\left(t^{\prime}\right) \mathrm{d} t^{\prime}+\widetilde{\theta}_{\rho}\right)}_{\widetilde{m}_{1}(t)} \\
& +\underbrace{\sqrt{\frac{\widetilde{\Omega}_{v}(t)}{\widetilde{K}(t)+1}} \sum_{n=1}^{N} \sqrt{\frac{1}{N}} \cos \left(2 \pi \int_{0}^{t} \tilde{f}_{n}\left(t^{\prime}\right) \mathrm{d} t^{\prime}+\widetilde{\theta}_{n}\right)}_{\tilde{\mu}_{1}(t)} \\
& \widetilde{v}_{2}(t) \\
& =\underbrace{\sqrt{\frac{\widetilde{K}(t) \widetilde{\Omega}_{v}(t)}{\widetilde{K}(t)+1}} \sin \left(2 \pi \int_{0}^{t} \tilde{f}_{\rho}\left(t^{\prime}\right) \mathrm{d} t^{\prime}+\widetilde{\theta}_{\rho}\right)}_{\widetilde{m}_{2}(t)} \\
& +\underbrace{\sqrt{\frac{\widetilde{\Omega}_{v}(t)}{\widetilde{K}(t)+1}} \sum_{n=1}^{N} \sqrt{\frac{1}{N}} \sin \left(2 \pi \int_{0}^{t} \widetilde{f}_{n}\left(t^{\prime}\right) \mathrm{d} t^{\prime}+\widetilde{\theta}_{n}\right)}_{\tilde{\mu}_{2}(t)} .
\end{aligned}
$$

In the following, the joint distribution of $\widetilde{v}_{1}(t)$ and $\widetilde{v}_{2}(t)$ can be expressed as $p_{\widetilde{v}_{1} \widetilde{v}_{2}}\left(t, z_{1}, z_{2}\right)=p_{\widetilde{v}_{1}}\left(t, z_{1}\right) p_{\widetilde{v}_{2}}\left(t, z_{2}\right)$, where $p_{\widetilde{v}_{i}}\left(t, z_{i}\right)$ denotes the PDF of $\widetilde{v}_{i}(t)(i=1,2)$ and can be calculated by

$$
p_{\widetilde{v}_{i}}\left(t, z_{i}\right)=p_{\widehat{\mu}_{i}}\left(t, z_{i}\right) p_{\bar{m}_{i}}\left(t, z_{i}\right) .
$$

According to the definition of $\widehat{m}_{i}(t)(i=1,2)$ in (14), the PDF of $\widehat{m}_{i}(t)$ can be expressed as

$$
p_{\widehat{m}_{i}}\left(t, z_{i}\right)=\delta\left(z_{i}-\widehat{m}_{i}(t)\right)
$$

Using $[25,(2.15)]$ and the relationship between the distribution and the characteristic function, the PDF of $\widehat{\mu}_{i}(t)(i=$ 1,2 ) can be proved as

$$
\begin{aligned}
& p_{\widehat{\mu}_{1}}\left(t, z_{1}\right)=\int_{-\infty}^{\infty} \Psi_{\widehat{\mu}_{1}}(t, x) e^{-j 2 \pi x z_{1}} \mathrm{~d} x \\
& =2 \int_{0}^{\infty}\left[\prod_{n=1}^{N} J_{0}\left(2 \pi x \sqrt{\frac{\widetilde{\Omega}_{v}(t)}{N(\widetilde{K}(t)+1)}}\right)\right] \\
& \cdot \cos \left(2 \pi x z_{1}\right) \mathrm{d} x .
\end{aligned}
$$

Substituting (16) and (17) into (15), we can obtain the joint distribution $p_{\widetilde{v}_{1} \widetilde{v}_{2}}\left(t, z_{1}, z_{2}\right)$ of two random variables $\widetilde{v}_{1}(t)$ and $\widetilde{v}_{2}(t)$.

Then, let us set $z_{1}=z \cos \theta$ and $z_{2}=z \sin \theta$ [25]; the time-variant joint PDF of the envelope $\widetilde{\xi}(t)=|\widetilde{v}(t)|$ and phase $\widetilde{\vartheta}(t)=\arg \{\widetilde{v}(t)\}$ can be derived as

$$
\begin{aligned}
& p_{\tilde{\xi} \widetilde{\vartheta}}(t, \theta, z)=z p_{\widetilde{v}_{1} \widetilde{v}_{2}}(t, z \cos \theta, z \sin \theta)=2 \pi z \int_{0}^{\infty}\left[\prod_{n=1}^{N} J_{0}\left(2 \pi x \sqrt{\frac{\widetilde{\Omega}_{v}(t)}{N(\widetilde{K}(t)+1)}}\right)\right] \\
& \cdot J_{0}\left(2 \pi x \sqrt{z^{2}+\frac{\widetilde{K}(t) \widetilde{\Omega}_{v}(t)}{\widetilde{K}(t)+1}-2 z \sqrt{\frac{\widetilde{K}(t) \widetilde{\Omega}_{v}(t)}{\widetilde{K}(t)+1}} \cos \left(\theta-2 \pi \int_{0}^{t} \widetilde{f}_{\rho}\left(t^{\prime}\right) \mathrm{d} t^{\prime}-\widetilde{\theta}_{\rho}\right)}\right) x \mathrm{~d} x,
\end{aligned}
$$

where $z \geq 0$ and $\theta \in(-\pi, \pi)$. Finally, $p_{\tilde{\xi}}(t, z)$ can be obtained by integrating (18) over $\theta$ with $[26,(6.684-1)]$ as

$$
\begin{aligned}
& p_{\tilde{\xi}}(t, z)=\int_{-\pi}^{\pi} p_{\tilde{\xi} \widetilde{\vartheta}}(t, \theta, z) \mathrm{d} \theta=(2 \pi)^{2} \\
& \quad \cdot z \int_{0}^{\infty}\left[\prod_{n=1}^{N} J_{0}\left(2 \pi x \sqrt{\frac{\widetilde{\Omega}_{v}(t)}{N(\widetilde{K}(t)+1)}}\right)\right] \\
& \quad \cdot J_{0}\left(2 \pi x \sqrt{\frac{\widetilde{K}(t) \widetilde{\Omega}_{v}(t)}{\widetilde{K}(t)+1}}\right) J_{0}(2 \pi z x) x \mathrm{~d} x, \quad z \geq 0 .
\end{aligned}
$$

Eq. (19) shows that $p_{\tilde{\xi}}(t, z)$ is completely determined by the parameters of $N, \widetilde{\Omega}_{v}(t)$, and $\widetilde{K}(t)$, whereas the frequency parameters have no influence. Since $\widetilde{\Omega}_{v}(t)$ and $\widetilde{K}(t)$ are fixed at any given time instant, by using $[26,(6.618-5)]$ one can demonstrate that the PDF tends to the theoretical Rice distribution as (2) with $N \rightarrow \infty$. Similarly, by integrating $p_{\tilde{\xi} \widetilde{\vartheta}}(t, \theta, z)$ over $z$ and using $[26,(6.52)]$, the instantaneous phase PDF at any time instant can be proved to tend to the reference $\mathrm{PDF}$ as (3).

4.2. ACF. The ACF of nonstationary channels is also no longer time-invariant as (5) and becomes a function of both time lag $\tau$ and time $t$. The time-variant ACF of (8) can be defined as

$$
\begin{aligned}
r_{\widetilde{v}}(t, \tau)= & E\left[\widetilde{v}(t) \widetilde{v}^{*}(t+\tau)\right] \\
= & \frac{\widetilde{\Omega}_{v}(t)}{\widetilde{K}(t)+1} r_{\widetilde{\mu} \widetilde{\mu}}(t, \tau) \\
& +\frac{\widetilde{K}(t) \widetilde{\Omega}_{v}(t)}{\widetilde{K}(t)+1} r_{\widetilde{m} \widetilde{m}}(t, \tau),
\end{aligned}
$$




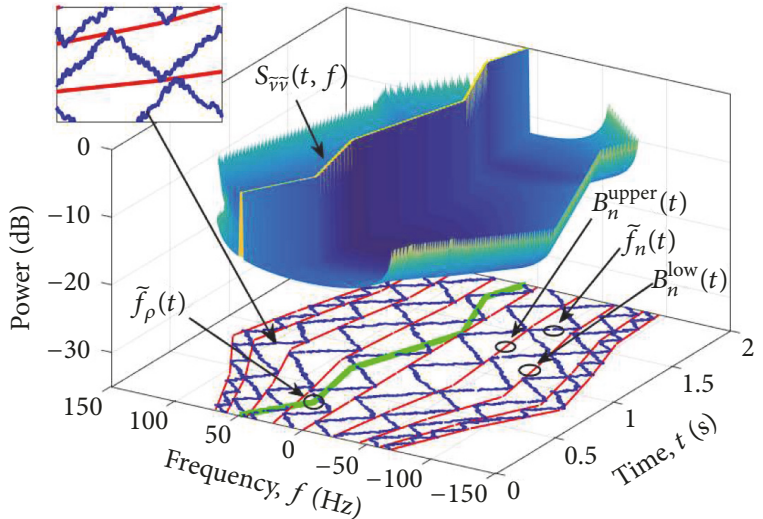

FIGURE 1: The computation method of frequency parameters.

where $r_{\widetilde{\mu} \widetilde{\mu}}(t, \tau)$ and $r_{\widetilde{m} \widetilde{m}}(t, \tau)$ denote the ACFs of the NLOS component and LOS component, respectively. In reality, the time lag range is much smaller than the updating interval $\left(\tau \ll T_{u}\right)$, and $r_{\widetilde{m} \widetilde{m}}(t, \tau)$ can be approximately calculated by

$$
r_{\widetilde{m} \widetilde{m}}(t, \tau)=e^{j 2 \pi \int_{t}^{t+\tau} \tilde{f}_{\rho}\left(t^{\prime}\right) \mathrm{d} t^{\prime}}=e^{j \pi \tau\left(\tilde{f}_{\rho}(t)+\tilde{f}_{\rho}(t+\tau)\right)} .
$$

The result in (21) clearly shows that the ACF of the LOS component changes over time. It reduces to the corresponding part of reference result as (5) when the frequency parameter $\widetilde{f}_{\rho}(t)$ is constant.
The time-variant ACF of the NLOS component with the VM distribution for AoAs can be expressed as follows:

$$
\begin{gathered}
r_{\tilde{\mu} \tilde{\mu}}(t, \tau)=\frac{1}{2 \pi I_{0}(\kappa)} \int_{-\pi}^{\pi} \sqrt{e^{\kappa \cos \left(\alpha_{n}(t)-\widetilde{m}_{\alpha}(t)\right)}} \\
\cdot \sqrt{e^{\kappa \cos \left(\alpha_{n}(t+\tau)-\widetilde{m}_{\alpha}(t+\tau)\right)}} e^{j 2 \pi \int_{t}^{t+\tau} \widetilde{f}_{n}\left(t^{\prime}\right) \mathrm{d} t^{\prime}} \mathrm{d} \alpha_{n} .
\end{gathered}
$$

The distribution factor $\kappa$ is almost unchanged during a short time interval, so $r_{\tilde{\mu} \tilde{\mu}}(t, \tau)$ can be simplified by using $\alpha_{n}(t+\tau)-$ $\widetilde{m}_{\alpha_{n}}(t+\tau) \approx \alpha_{n}(t)-\widetilde{m}_{\alpha_{n}}(t)$ as

$$
\begin{aligned}
& r_{\widetilde{\mu} \tilde{\mu}}(t, \tau)=\frac{1}{2 \pi I_{0}(\kappa)} \int_{-\pi}^{\pi} e^{\kappa \cos \left(\alpha_{n}(t)-\widetilde{m}_{\alpha}(t)\right)} \\
& \cdot e^{j 2 \pi \int_{t}^{t+\tau} f_{\max } \cos \left(\alpha_{n}\left(t^{\prime}\right)\right) \mathrm{d} t^{\prime}} \mathrm{d} \alpha_{n}=\frac{1}{2 \pi I_{0}(\kappa)} \\
& \cdot \int_{-\pi}^{\pi} e^{\kappa \cos \left(\alpha_{n}(t)-\widetilde{m}_{\alpha}(t)\right)} \\
& \cdot e^{j \pi f_{\max } \tau\left(\cos \left(\alpha_{n}(t+\tau)\right)+\cos \left(\alpha_{n}(t)\right)\right)} \mathrm{d} \alpha_{n},
\end{aligned}
$$

where $\widetilde{m}_{\alpha_{n}}(t)$ denotes the mean angle of AoAs. Setting and substituting $\Delta_{n}(t, \tau)=\alpha_{n}(t+\tau)-\alpha_{n}(t)$ into (23), it yields

$$
\begin{aligned}
& r_{\tilde{\mu} \tilde{\mu}}(t, \tau)=\frac{1}{2 \pi I_{0}(\kappa)} \int_{-\pi}^{\pi} e^{\kappa \cos \left(\alpha_{n}(t)-\widetilde{m}_{\alpha}(t)\right)} \\
& \cdot e^{j 2 \pi f_{\max } \tau \cos \left(\Delta_{n}(t, \tau) / 2\right) \cos \left(\alpha_{n}(t)+\Delta_{n}(t, \tau) / 2\right)} \mathrm{d} \alpha_{n} .
\end{aligned}
$$

With the help of $[26,(3.338-4)]$, the closed-form solution of $r_{\widetilde{\mu} \tilde{\mu}}(t, \tau)$ is given as

$$
r_{\tilde{\mu} \tilde{\mu}}(t, \tau)=\frac{I_{0}\left(\sqrt{\kappa^{2}+j 2 \pi \kappa f_{\max } \tau\left(\cos \left(\widetilde{m}_{\alpha_{n}}(t)\right)+\cos \left(\widetilde{m}_{\alpha_{n}}(t)-\Delta_{n}(t, \tau)\right)\right)-2\left(\pi f_{\max } \tau\right)^{2}\left(\cos \left(\Delta_{n}(t, \tau)\right)+1\right)}\right)}{I_{0}(\kappa)} .
$$

Finally, the theoretical ACF $r_{\widetilde{v v}}(t, \tau)$ of the proposed simulator can be obtained by substituting (21) and (25) into (20).

4.3. DPSD. The time-variant DPSD of (8) can be defined as

$$
\begin{aligned}
S_{\widetilde{v v}}(t, f)= & \frac{\widetilde{\Omega}_{v}(t)}{\widetilde{K}(t)+1} S_{\widetilde{\mu} \widetilde{\mu}}(t, f) \\
& +\frac{\widetilde{K}(t) \widetilde{\Omega}_{v}(t)}{\widetilde{K}(t)+1} S_{\widetilde{m} \widetilde{m}}(t, f) .
\end{aligned}
$$

Combining with (10), the DPSD of LOS component can be calculated as

$$
\begin{aligned}
& S_{\widetilde{m} \widetilde{m}}(t, f) \\
& \quad=\delta\left(f-\widetilde{F}_{\rho}^{l}-\frac{\widetilde{F}_{\rho}^{l+1}-\widetilde{F}_{\rho}^{l}}{T_{u}}\left[t-(l-1) T_{u}\right]\right) .
\end{aligned}
$$

Base on (11), we can obtain the instantaneous phase caused by the time-variant frequency of NLOS component as

$$
\begin{aligned}
\tilde{\phi}_{n}(t) & =2 \pi \int_{0}^{t} \tilde{f}_{n}(x) \mathrm{d} x \\
& =2 \pi\left(b_{n}^{l} t-(l-1) k_{n}^{l} T_{u} t+\frac{k_{n}^{l}}{2} t^{2}\right) .
\end{aligned}
$$

Substituting (28) into (8), the NLOS component can be rewritten as

$$
\tilde{\mu}(t)=\sum_{n=1}^{N} \sqrt{\frac{1}{N}} e^{j\left[2 \pi\left(b_{n}^{l} t-(l-1) k_{n}^{l} T_{u} t+\left(k_{n}^{l} / 2\right) t^{2}\right)+\tilde{\theta}_{n}\right]} .
$$


With the help of the Wigner-Ville distribution [19, 27], the DPSD of (29) can be expressed as

$$
\begin{aligned}
& W_{\tilde{\mu} \tilde{\mu}}(t, f) \\
& =\sum_{n=1}^{N} \frac{1}{N} \delta\left(f-\left(b_{n}^{l}-(l-1) k_{n}^{l} T_{u}+k_{n}^{l}\right) t\right) \\
& \quad+4 \sum_{n=1}^{N-1} \sum_{m=2, m>n}^{N} \frac{1}{\sqrt{\beta_{n m}}} \cos \left(\frac{\pi}{4}+\alpha_{n m}-\frac{4 \pi \gamma_{n m}^{2}}{\beta_{n m}}\right),
\end{aligned}
$$

where

$$
\begin{aligned}
\alpha_{n m}= & \tilde{\theta}_{n}-\tilde{\theta}_{m}+\pi\left(k_{n}^{l}-k_{m}^{l}\right) t^{2} \\
& +2 \pi\left(b_{n}^{l}-b_{m}^{l}-(l-1)\left(k_{n}^{l}-k_{m}^{l}\right) T_{u}\right) t \\
\beta_{n m}= & \left|k_{n}^{l}-k_{m}^{l}\right| \\
\gamma_{n m}= & f-\frac{b_{n}^{l}+b_{m}^{l}-(l-1)\left(k_{n}^{l}+k_{m}^{l}\right) T_{u}}{2}-\frac{k_{n}^{l}+k_{m}^{l}}{2} t .
\end{aligned}
$$

The initial phase is random and uniformly distributed over $(0,2 \pi]$, so the second term in (30) can be removed by averaging over the phases. Therefore, the DPSD of NLOS component can be rewritten as

$$
\begin{aligned}
S_{\widetilde{\mu} \tilde{\mu}}(t, f) & =\left.W_{\widetilde{\mu} \widetilde{\mu}}(t, f)\right|_{\tilde{\theta}_{n}} \\
& =\sum_{n=1}^{N} \frac{1}{N} \delta\left(f-\left(b_{n}^{l}-(l-1) k_{n}^{l} T_{u}+k_{n}^{l}\right) t\right) .
\end{aligned}
$$

Finally, substituting (27) and (32) into (26), the theoretical DPSD $S_{\widetilde{v v}}(t, f)$ of the proposed simulator can be obtained.

\section{Simulation and Validation}

We validate the proposed simulator in a typical downlink channel of the mobile communication systems. The base station (BS) and MS are both equipped with normalized omnidirectional antennas, the carrier frequency is $2.4 \mathrm{GHz}$, and the AoAs follow the VM distribution with $\kappa=3$. The initial distance between the BS and MS is $140 \mathrm{~m}$, and the MS moves away from the BS with the angle of $\theta=45^{\circ}$ and the speed of $v=20 \mathrm{~m} / \mathrm{s}$. It is also assumed that the LOS and NLOS components exist during the simulation.

The simulator is configured as $N=32, N_{s f}=10$, and $T_{u}=20 \mathrm{~ms}$. The generated channel phase, fading envelope, and Doppler frequency within $2 \mathrm{~s}$ are given in Figure 2. For comparison purpose, the corresponding output results of the simulators in $[10,11,13,14]$ and the theoretical Doppler frequency are also given in the figure. It shows that the output phases of two kinds of simulators are different, which results in the difference of channel envelopes. Moreover, the Doppler frequency of the proposed simulator is much closer to the theoretical one. Submitting $N=32$ and other channel parameters into (19), the theoretical PDF can be obtained and given in Figure 3. It is clearly showed that the PDF changes
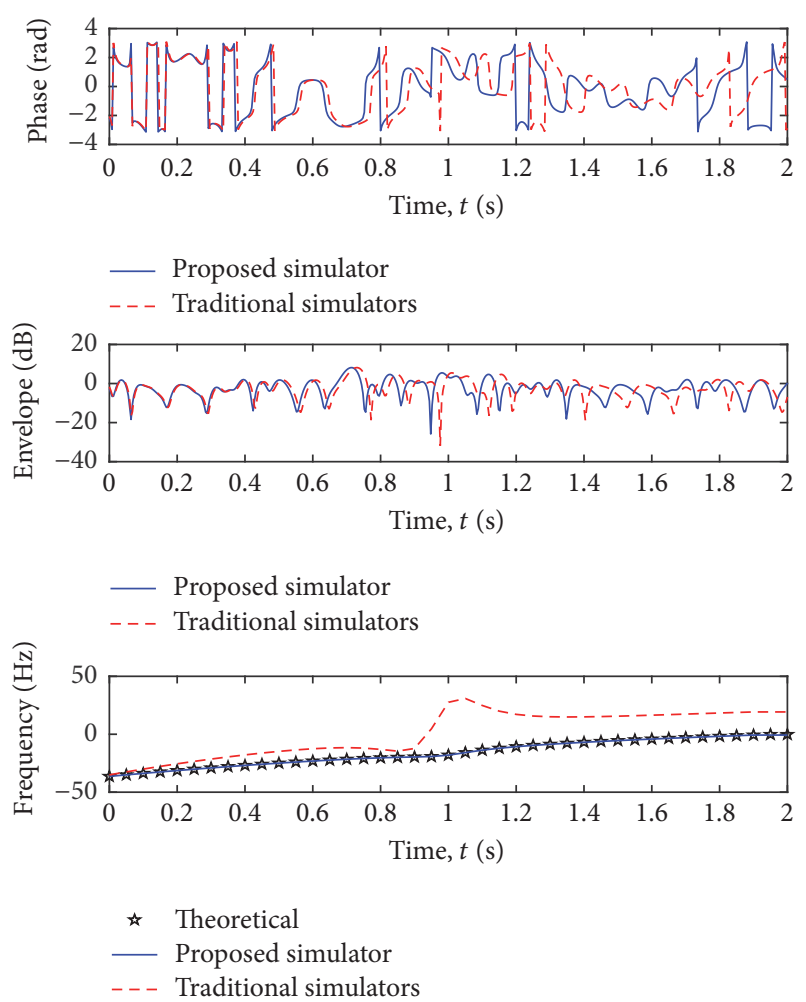

FIgURE 2: Output channel fading phases, envelopes, and Doppler frequencies of the proposed simulator and traditional simulators in $[10,11,13,14]$.

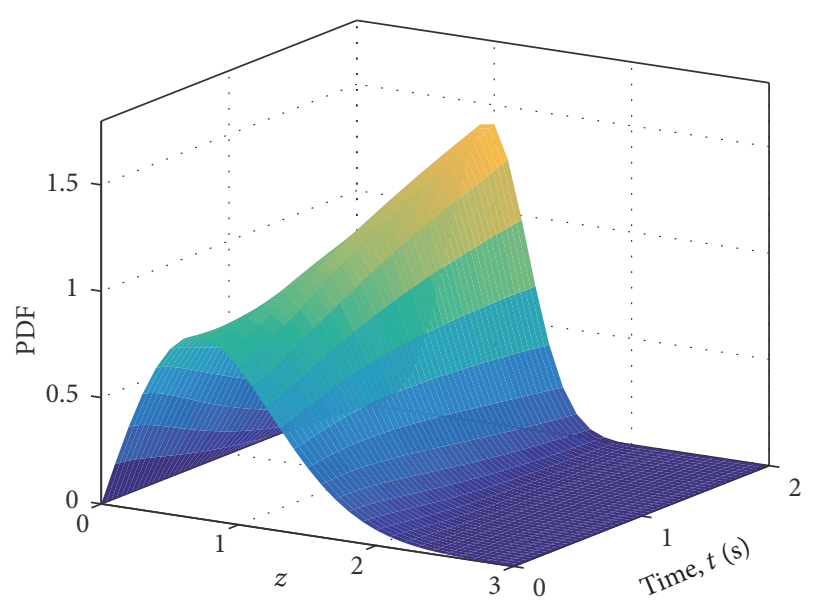

FIgURE 3: Time-variant theoretical PDF of channel fading envelope $(N=32)$.

over time due to the time-variant channel parameters. In addition, the output PDFs of the proposed simulator at three time instants $t=0 \mathrm{~s}, 1 \mathrm{~s}$ and $2 \mathrm{~s}$ are compared with the corresponding theoretical results in Figure 4. The reference PDFs which can be viewed as the theoretical results with $N=$ $\infty$ are also given in Figure 4. It is showed that the simulated results match well with the corresponding theoretical and reference ones, which verifies the theoretical derivations as well as the simulator. 


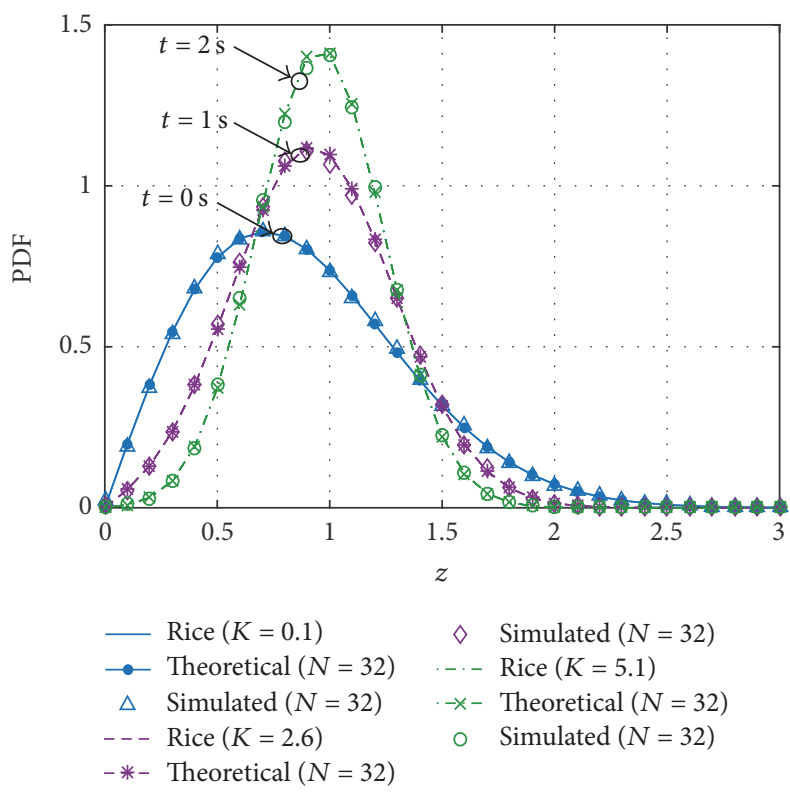

FIgURE 4: Theoretical and simulated PDFs of fading envelopes at different time instants $\left(f_{c}=2.4 \mathrm{GHz}, \kappa=3, D_{0}=140 \mathrm{~m}, \theta=45^{\circ}\right.$, $v=20 \mathrm{~m} / \mathrm{s}, N_{s f}=10, N=32$, and $\left.T_{u}=20 \mathrm{~ms}\right)$.

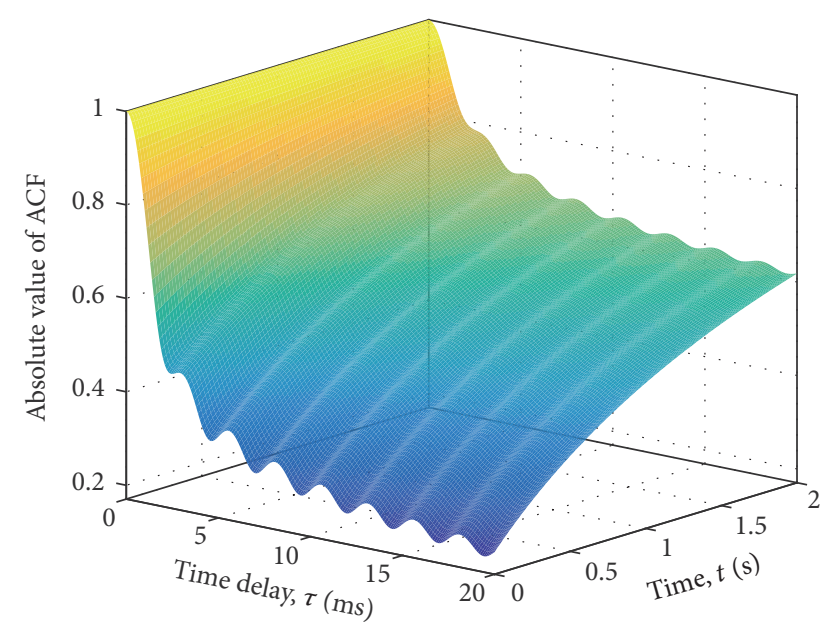

FIGURE 5: Absolute values of the time-variant theoretical ACF $\left(f_{c}=\right.$ $2.4 \mathrm{GHz}, \kappa=3, D_{0}=140 \mathrm{~m}, \theta=45^{\circ}$, and $v=20 \mathrm{~m} / \mathrm{s}$ ).

Under the same condition, by using (20), the absolute value of the theoretical ACF is calculated and given in Figure 5. For comparison purpose, the theoretical ACFs at three time instants $t=0 \mathrm{~s}, 1 \mathrm{~s}$, and $2 \mathrm{~s}$ are extracted from Figure 5 and the corresponding simulated ACFs are shown in Figure 6. Similarly, the theoretical DPSDs calculated by (26) are given in Figure 7(a) and the simulation results are given in Figure 7(b). Figure 7 clearly demonstrates the timevariety of the LOS component and NLOS components along the trajectory of the MS. It also can be seen that Figure 7 has an asymmetric DPSD, which is the consequence of the nonisotropic scattering condition. In addition, the trends and

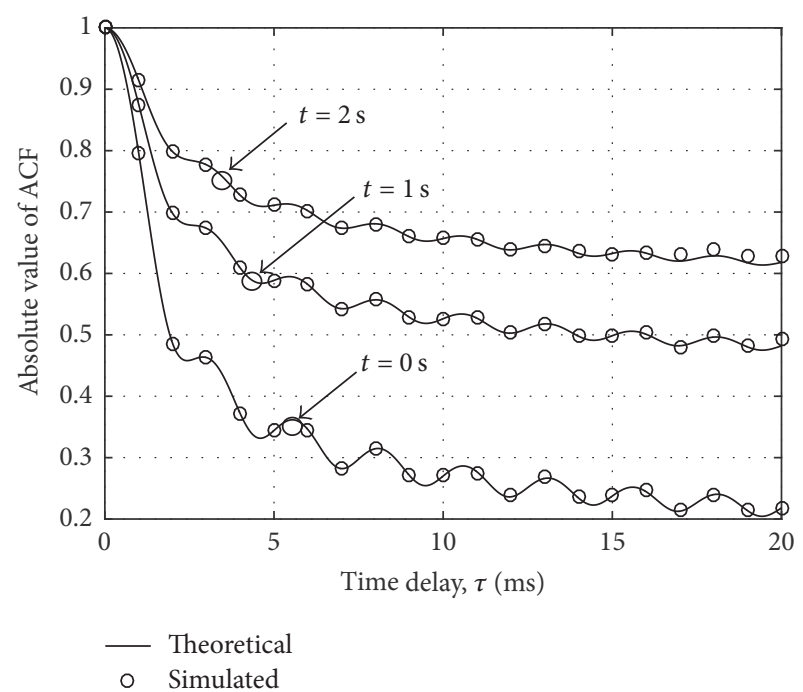

FIgURE 6: Absolute values of theoretical and simulated ACF at different time instants $\left(f_{c}=2.4 \mathrm{GHz}, \kappa=3, D_{0}=140 \mathrm{~m}, \theta=45^{\circ}\right.$, $v=20 \mathrm{~m} / \mathrm{s}, N_{s f}=10, N=32$, and $T_{u}=20 \mathrm{~ms}$ ).

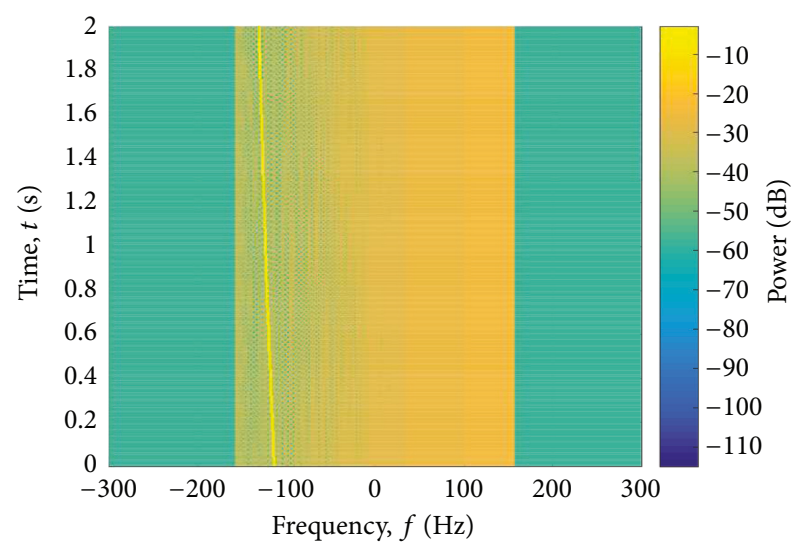

(a)

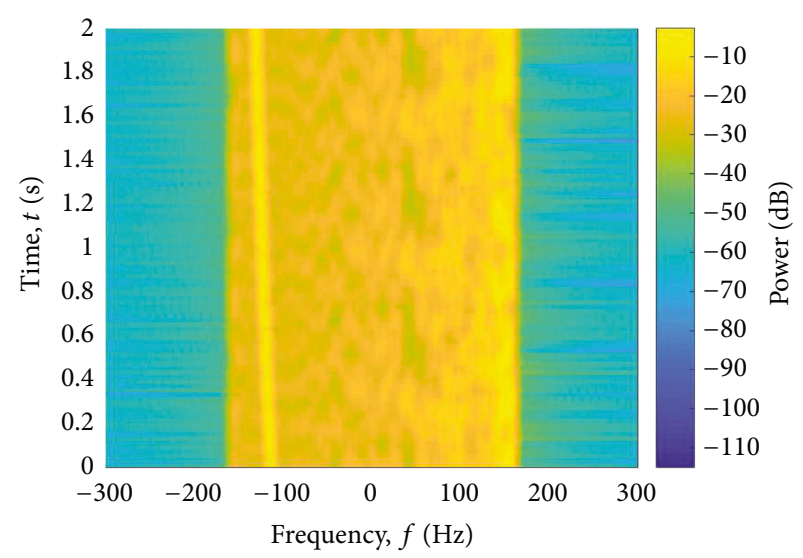

(b)

FIgURE 7: (a) Theoretical and (b) simulated time-variant DPSDs $\left(f_{c}=2.4 \mathrm{GHz}, \kappa=3, D_{0}=140 \mathrm{~m}, \theta=45^{\circ}, v=20 \mathrm{~m} / \mathrm{s}, N_{s f}=10\right.$, $N=32$, and $T_{u}=20 \mathrm{~ms}$ ). 
shapes of theoretical and simulated DPSDs are very similar, which verifies the correctness of both the simulator and derivations. Finally, the good agreements of theoretical and simulated results in Figures 6 and 7 confirm the derivations of (25) and (32), as well as the proposed simulator.

\section{Conclusion}

Further research on the simple and efficient nonstationary channel simulators is highly desirable for the development and performance evaluation of future wireless communication systems. In this paper, we have presented a new simulator to reproduce the nonstationary Rice fading channels under nonisotropic scattering scenarios. The proposed simulator can also be applied to simulate Rayleigh fading channels by adjusting channel parameters. The computation methods of time-variant simulation parameters, that is, initial phases and frequencies of the LOS component and NLOS component, have been analyzed and given in detail. Based on the new simulator, the theoretical expressions for the PDFs of envelope and phase, ACF, and DPSD under VM distribution scattering environment have also been derived. Finally, the simulation results have demonstrated that our proposed simulator can reproduce the nonstationary fading channels with accurate statistical properties such as PDF, ACF, and DPSD.

\section{Conflicts of Interest}

The authors declare that they have no conflicts of interest.

\section{Acknowledgments}

This work is supported by Fundamental Research Funds for the Central Universities (Grant no. NS2015046).

\section{References}

[1] R. H. Clarke, "A statistical theory of mobile-radio reception," Bell Labs Technical Journal, vol. 47, no. 6, pp. 957-1000, 1968.

[2] W. C. Jakes, Microwave Mobile Communications, Wiley-IEEE Press, Piscataway, NJ, USA, 2nd edition, 1994.

[3] M. Pätzold, U. Killat, Y. Li, and F. Laue, "Modeling, analysis, and simulation of nonfrequency-selective mobile radio channels with asymmetrical Doppler power spectral density shapes," IEEE Transactions on Vehicular Technology, vol. 46, no. 2, pp. 494-507, 1997.

[4] C.-X. Wang, M. Pätzold, and D. Yuan, "Accurate and efficient simulation of multiple uncorrelated Rayleigh fading waveforms," IEEE Transactions on Wireless Communications, vol. 6, no. 3, pp. 833-839, 2007.

[5] C. Xiao, Y. R. Zheng, and N. C. Beaulieu, "Novel sum-ofsinusoids simulation models for Rayleigh and Rician fading channels," IEEE Transactions on Wireless Communications, vol. 5, no. 12, pp. 3667-3679, 2006.

[6] M. Pätzold, C.-X. Wang, and B. O. Hogstad, "Two new sum-ofsinusoids-based methods for the efficient generation of multiple uncorrelated rayleigh fading waveforms," IEEE Transactions on Wireless Communications, vol. 8, no. 6, pp. 3122-3131, 2009.
[7] A. G. Zajić and G. L. Stüber, "Efficient simulation of rayleigh fading with enhanced de-correlation properties," IEEE Transactions on Wireless Communications, vol. 5, no. 7, pp. 1866-1875, 2006.

[8] C. A. Gutiérrez and M. Pätzold, “The design of sum-of-cisoids rayleigh fading channel simulators assuming non-isotropic scattering conditions," IEEE Transactions on Wireless Communications, vol. 9, no. 4, pp. 1308-1314, 2010.

[9] J. Meinila, P. Kyosti, L. Hentila et al., "WINNER+ final channel models," 2010, http://projects.celtic-initiative.org/winner+/.

[10] A. Ghazal, C.-X. Wang, B. Ai, D. Yuan, and H. Haas, "A nonstationary wideband MIMO channel model for highmobility intelligent transportation systems," IEEE Transactions on Intelligent Transportation Systems, vol. 16, no. 2, pp. 885-897, 2015.

[11] Y. Yuan, C.-X. Wang, Y. He, M. M. Alwakeel, and E.-H. M. Aggoune, "3D Wideband Non-Stationary Geometry-Based Stochastic Models for Non-Isotropic MIMO Vehicle-to-Vehicle Channels," IEEE Transactions on Wireless Communications, vol. 14, no. 12, pp. 6883-6895, 2015.

[12] A. Borhani, G. L. Stuber, and M. Patzold, "A random trajectory approach for the development of nonstationary channel models capturing different scales of fading," IEEE Transactions on Vehicular Technology, vol. 66, no. 1, pp. 2-14, 2016.

[13] A. Ghaza, Y. Yuan, and C.-X. Wang, "A non-stationary IMT-A MIMO channel model for high-mobility wireless communication systems," IEEE Trans. Wireless Commun, vol. 16, no. 4, pp. 2057-2068, 2017.

[14] J. Bian, J. Sun, C. Wang et al., "A WINNER+ based 3-D nonstationary wideband MIMO channel model," IEEE Transactions on Wireless Communications, 2017.

[15] Q. Zhu, K. Jiang, X. Chen, C.-X. Wang, X. Hu, and Y. Yang, "A modified non-stationary MIMO channel model under 3D scattering scenarios," in Proceedings of the IEEE/CIC International Conf. on IEEE, pp. 1-5, Qingdao, China, 2017.

[16] W. Dahech, M. Pätzold, C. A. Gutiérrez, and N. Youssef, "A Non-Stationary Mobile-to-Mobile Channel Model Allowing for Velocity and Trajectory Variations of the Mobile Stations," IEEE Transactions on Wireless Communications, vol. 16, no. 3, pp. 1987-2000, 2017.

[17] X. Liu, Q. Zhu, X. Chen, and K. Jiang, "A new simulation model for non-stationary fading channel," in Proceedings of the 3rd International Conference on Electronic Design, ICED 2016, pp. 66-69, tha, August 2016.

[18] Q. Zhu, X. Liu, X. Yin, X. Chen, and C. Xue, "A novel simulator of nonstationary random mimo channels in rayleigh fading scenarios," International Journal of Antennas and Propagation, vol. 2016, Article ID 3492591, 2016.

[19] M. Patzold and C. A. Gutierrez, "The wigner distribution of sum-of-cissoids and sum-of-chirps processes for the modelling of stationary and non-stationary mobile channels," in Proceedings of the 83rd IEEE Vehicular Technology Conference, VTC Spring 2016, pp. 1-5, Nanjing, China, May 2016.

[20] B. Chen, Z. Zhong, and B. Ai, "Stationarity intervals of timevariant channel in high speed railway scenario," China Communications, vol. 9, no. 8, pp. 64-70, 2012.

[21] B. Hogstad, M. Patzold, N. Youssef, and . Dongwoo Kim, "A MIMO mobile-to-mobile channel model: Part II-The simulation model," in Proceedings of the 16th IEEE PIMRC 2005, pp. 562-567, Berlin, Germany, 2005.

[22] C. A. Gutiérrez and M. Pätzold, "The generalized method of equal areas for the design of sum-of-cisoids simulators 
for mobile Rayleigh fading channels with arbitrary Doppler spectra," Wireless Communications and Mobile Computing, vol. 13, no. 10, pp. 951-966, 2013.

[23] M. Patzold, Mobile Fading Channels, John Wiley Sons, Chichester, UK, 2002.

[24] T. A. Thomas, F. W. Vook, E. Visotsky, E. Mellios, G. S. Hilton, and A. R. Nix, "3D extension of the 3GPP/ITU channel model," in Proceedings of the 2013 IEEE 77th Vehicular Technology Conference, VTC Spring 2013, Dresden, Germany, June 2013.

[25] A. Papoulis and S. Pillai, Probability, Random Variables, and Stochastic Processes, McGraw-Hill, New York, NY, USA, 4th edition, 2002.

[26] I. S. Gradshteyn and I. M. Ryzhik, Table of Integrals, Series, and Products, Academic press, 2014.

[27] A. Bruscato and C. M. Toloi, "Spectral analysis of nonstationary processes using the Fourier transform," Brazilian Journal of Probability and Statistics, vol. 18, no. 1, pp. 69-102, 2004. 


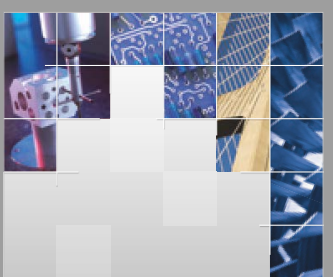

\section{Enfincering}
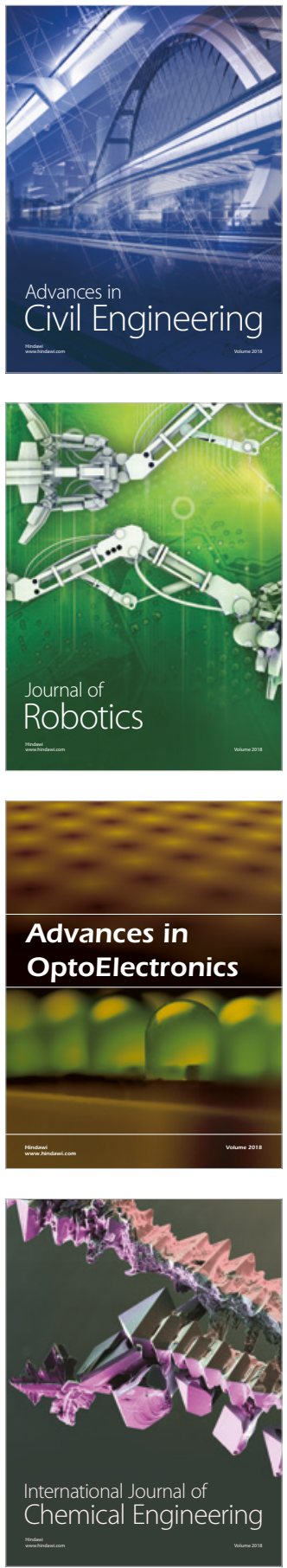

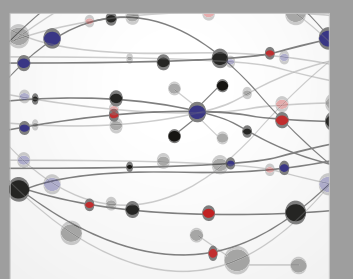

\section{Rotating \\ Machinery}

The Scientific World Journal

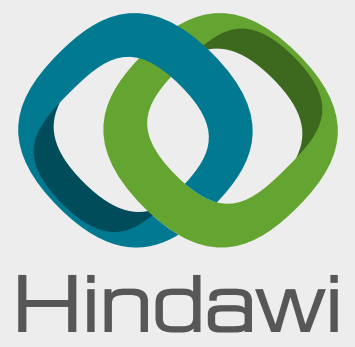

Submit your manuscripts at

www.hindawi.com
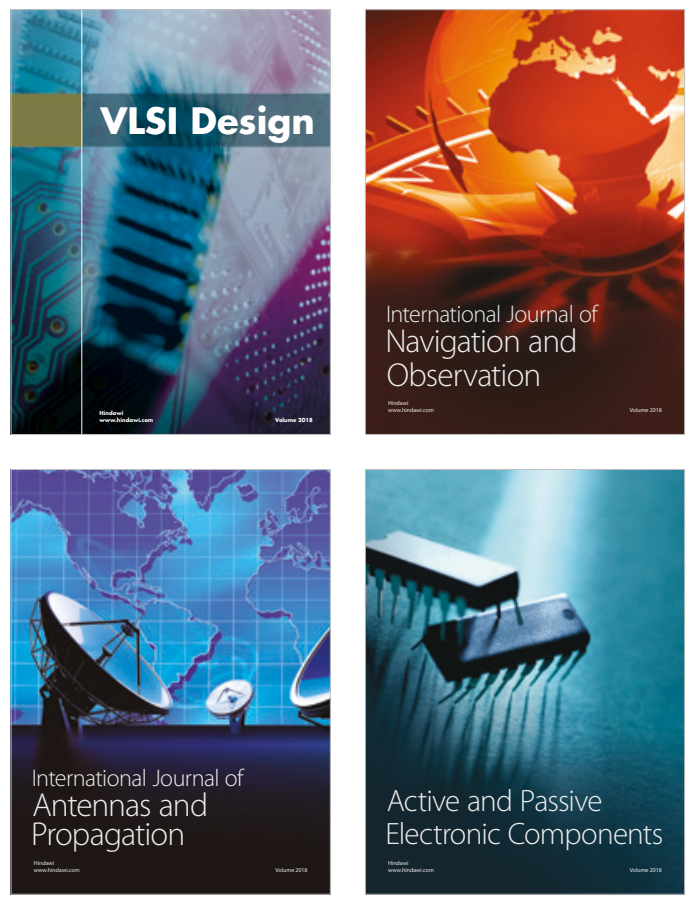
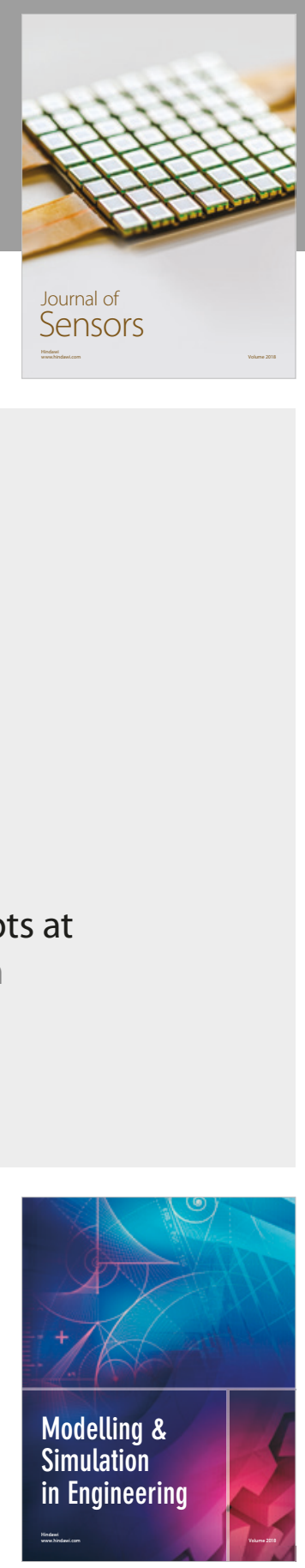

\section{Advances \\ Multimedia}
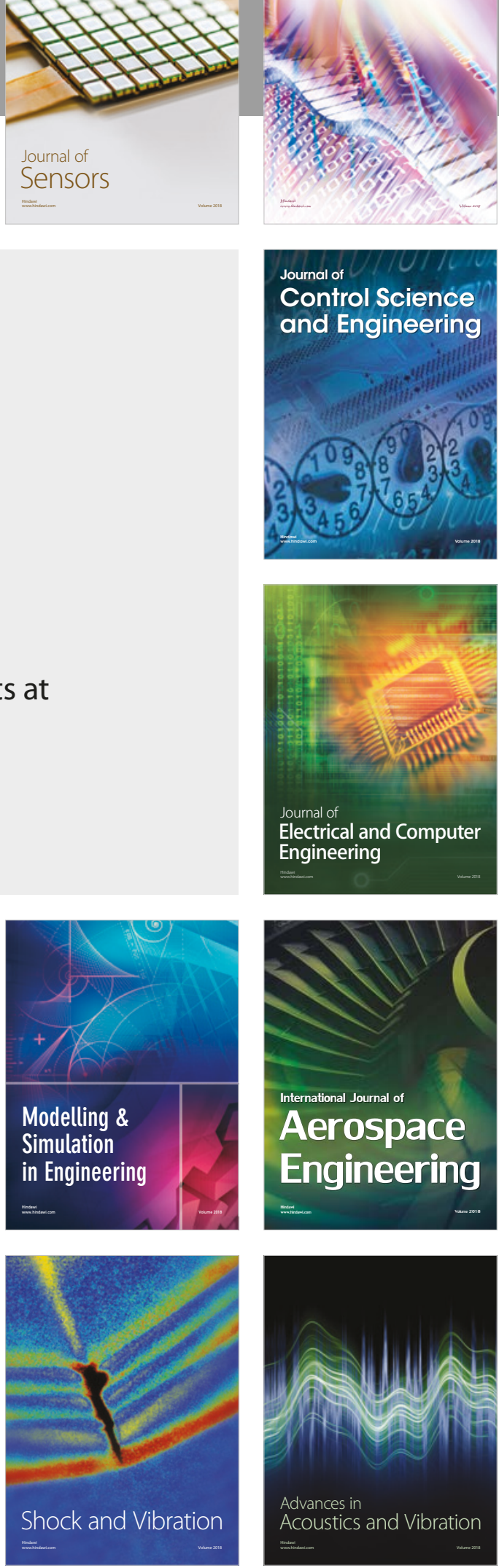